\title{
ALTERAÇÕES DIMENSIONAIS EM \\ PASTAS DE ÓXIDO DE ZINCO/EUGENOL: \\ UM NOVO MÉTODO PARA SUA MENSURAÇÃO
}

* Prof. Léo Werner Süffert

** Prof. Ennio Pessôa

SüFFERT, Léo Werner \& PESSÕA, Ennio. Alterações dimensionais em pastas de óxido de zinco/eugenol: um novo método para sua mensuração. Revista da Faculdade de Odontologra, Porto Alegre, 18/19/20: 39-55, $1976 / 8$.

Descritores: PASTAS DE ÓXIDO DE ZINCO/EUGENOL, ALTERAÇŏES DIMENSIONAIS

MATERIAIS ODONTOLÓGICOS - MOLDAGEM

\section{RESUMO}

Após um levantamento bibliográfico à respeito de pastas zincoenólicas e, selecionando os trabalho mais representativos, realizamos ensaios de altorações dimensionais com 4 marcas das referidas pastas, das mais utilizadas pelo cirúrgião dentista brasileiro.

Segundo informacões pessoais de vários investigadores citados em nossa bibliografia, trata se de uma propriedade dificil de verificar com precisão, dadas as características de muita viscosidade o pegajosidade destes materiais.

Por este motivo ntilizamos um método diferente, idealizado em nosso laheratório, para verifileação de alterações dimensionais de pastas zincoenólicas.

Os resultados que apresentamos através dos diversos gráficos e tabelas que constam do presente trabalho, demonstram alterações dimensionais desde $0,003 \%$ até $0,102 \%$, valores estes que, provarelmente, possam ser considerados negligenciaveis scb o ponto de vista clínico.

O que nos chamou a atenção, no entanto, foram os elevados valores de desvio padrão e variância, indicativos de elevada variabilidade dentro dos diversos ensaios.

O método utilizado já havia, entanto, comprovado sua alta reprodutibllidade quando utilizado na verifica-

* Professor Titular de Materiais Dentários da Faculdade de Odontologia/UFRGS

* Professor Adjunto de Materlals Dentários da Faculdade de Odontologla/UFRGS 
gão de alteraçoes dimensionais de mercaptanas e siliconas.

Uma hipótese para o fato aqui ver rificado (e que pretendemos pesquisar numa próxima investigação), é a d. que, durante armazenagem destes materiais, acondicionados à semelhança do dentiirrícios, poderá estar ocorrendo a sedimentação dos componentes de maior densidade.

Consequetemente havería o desiquilíbrio na composição, inobstante o método utilizado para estabelecer a proporção entre as duas pastas, tan10 por pêso, como por medida de comprimento, acarretando isto uma variabilidade de composição em cada misttura.

\section{INIRODUCก̃O}

$\mathrm{Na}$ moldagem de próteses totais e mesmo, no que se refere à parte mu. co-suportada, nas próteses parciais, à partir de 1930, as Pastas de ZnOEugenol passaram a ter cada vez mais aceitação de parte dos cirur. giões.dentistas.

Sua utilização ficou um pouco re. duzida com o advento das mercaptanas, siliconas e poliéteres, mas nem por isso deixam de apresentar excelentes resultados, quando utilizadas adequadamente, nos procedimentos e técnicas indicados.

Éstas pastas de um óxido metálico, são geralmente baseadas em combi. nações de óxido de Zinco em Euge. nól. As formulas dos produtos comerciais não tem sido publicadas, en. tretanto Ross ${ }^{13}$, sugeriu uma fórmu. la dentre as várias combinacões que experimentara em 1934, e que é a se. guinte: 85 partes de ZnO e 15 par- tes de colofônia, constituindo o pó. o líquido, por sua vez, era constitui. do por $60 \%$ de ugenól, 35\% de Bal. samo de Canadá e 5\% de Balsamo do Per'í.

Várias propriedades e característi. cas das muitas marcas comerciais destes produtos, que surgiram no mercado de artigos dentários à partir de então, foram pesquisadas, por diferentes pesquisadores, em diferen. tes épocas e, é importante que se re. gistre, com alguma freqüência, por métodos e equipamentos algo distintos.

Além disso, nem sempre pode per. ceber na propriedade pesquisada e, particularmente, na maneira como es. tá sendo pesquisada em laboratório, correlação ou relevância clínica da mesmas.

As características pesquisadas ini. cialmente, diziam respeito à compo. sição e natureza fundamental de rea. cões entre o óxido de zinco e o eu. genól, seus principais componentes.

Vários pesquisadores abordaram as pastas zincoenólicas sob este aspec. to, alguns deles relacionando modifi. cações de composição com propriedades de endurecimento. $3,5,9,13$, 14,19 .

Até o surgimento de Especifica. çoes, de parte da American Dental Association 4 , aliás, a preocupação dos investigadores se relacionava com reações e propriedades de endureci. mento tais como consistência de mis. tura, consistência no momento da moldagem propriamente dito, tempo de permanencia na bôca para poste. rior remocão etc.

Antes da $1^{\circ}$ revisão da Especifica. ção Nọ 16 da A.D.A. ${ }^{4}$, um dos requi. 
sitos estipulados era o de que não houvesse mais do que $17 \%$ de Eugenól presente na composição. ${ }^{12}$ Pre sentemente não existe esta limitação, bastando, no entanto, que o fabri. cante indique na embalagem conteú. do e seu percentual de Eugenól.

Algumas pesquisas ${ }^{2}, 6,10$ foram rea. lizadas, com o objetivo de fornecerem subsidios para uma eventual Especificação para estes materiais, o que logo se concretizou. ${ }^{4}$

Julgava.se importante conhecer o grau de viscosidade destes materiais no momento em que fossem levados em contato com os tecidos bucais pa. ra a moldagem, quando então, de a. cordo com a maior ou menor flacidês dos referidos tecidos, bem como, de acordo com a maior ou menor viscosidade do material, podería ocorrer mais ou menos deslocamento de tecidos.

o ensaio laboratorial que objetiva. va dar esta informacão foi denomina. do: Ensaio de Consistência e foi realizado por muitos pesquisadores. 1,2, $6,7,10,12,15,16,17,19$

Outro fator importante, dizia respeito às várias etapas de aumento de viscosidade, face a reacões químicas ${ }^{3}, 9$, informando ao cirurgião. dentista quanto tempo ele dispunha para manipular o material, inserí-1o na moldeira e posicioná-lo "in situ", na boca do paciente.

De outro lado, era importante tam. bém, saber quanto tempo o material deveria permanecer, imóvel, na boca $d_{0}$ paciente, antes que pudesse ser removido com segurança.

A obtenção destas informacões foi tentada de várias maneiras, procurando os investigadores, com maior ou menor sucesso, ensaios laborato. riais que se relacionassem com a si. tuação clínica na utilização do mate. rial.

Alguns utilizaram com este objetivo, a agulha de Gillmore de $1 / 4$ de libra e obtinham o que se convencionou chamar de Presa Inicial e Presa Final. 1, 2, 6, 9, 10, 16

Outros utilizavam, neste sentido, a agulha de Vicat. $7,12,14,15,18$

outros ainda, utilizaram o Pene. trômetro de Krebs ${ }^{5}$, como o recomendou posteriormente a Especificacão. ${ }^{4}$

A Presa Inicial, nos dias atuais e, segundo as referidas normas 4 , tem sido verificada através de uma barra cilindrica de $2,4 \mathrm{~mm}$ de diâmetro e pesando 10 gramas. ${ }^{12}$

Ao término da reação química e, dadas as características de pastas zincoenólicas, este material pode se apresentar num maior ou menor es. tado de rigidez.

O ensaio laboratorial que objetiva dar esta informação foi realizado mediante a agulha de Vicat ${ }^{15}$, inicial. mente, sendo substituido, após a aceitação das Especificações ${ }^{4}$, pelo $\mathrm{Pe}$ netrômetro de Krebs. 10, 12, 16.

Todo material de moldagem, para ser eficiente, deve ser capaz de re. produzir os mínimos detalhes anatô. micos existentes na boca e, neste sentido, vários investigadores tentaram ensaios laboratoriais que forne. cessem esta informação. 1, 2, 6, 10.

As resistências à compressãol e à tração 6,7 também foram motivos de estudo.

Mais recentemente, a idéia inicial de Wilson ${ }^{20}, 17$ tem obtido cada vez mais aceitação, para mensurar, com enorme precisão, as diversas etapas 
de endurecimento da pastas zinco. enólicas.

Wilson utiliza o que denominou de "Reômetro»», uma adaptação de um aparelho utilizado para medir a velo. cidade de polimerização de borrachas. O método do "Reômetro", já aceito nas Especificações Britânicas é, em nosso entender, aquele que, além de maior precisão, nos fornece informações, através de ensaios labo. ratoriais, de maior correlação com a situação clínica e de suma relevân. cia.

Apenas duas pesquisas 15, 17, den. tre as muitas que compulsamos e das quais fazemos constar apenas $20 \mathrm{em}$ nossas referencias blbliográficas, pro. curaram verificar ALTERAÇőES DI. MENSIONAIS destes materiais, de. correntes de suas reações químicas.

Segundo informacões obtidas de muitos investigadores, cujos traba. lhos citamos, trata.se de uma pro. priedade, "possivelmente pouco rele. vante, mas definitivamente muito di. fícil de verificar", dadas as caracte. rísticas de muita viscosidade e pega. josidade do material!

o presente trabalho tem, precisa. mente, como objetivo principal, mensurar as alterações dimensionais de algumas pastas de Zn0.Eugenól, por um método diferente e de maior re. produtividade, do que as até então su. geridas.

\section{MATERIAIS E METODOS}

\subsection{MATRIZ PARA ELABORACT̃O DOS CORPOS DE PROVA}

O conjunto de peças que compu. nha a matriz para elaboração dos cor. pos de prova, era constituido de 5 partes desmontáveis, A, B, C, D e E, conforme se pode observar na Fig. de $n^{\circ} 1$

"A" = Uma base retangular de acrílico (30 mm de largura por 60 $\mathrm{mm}$ de comprimento e $3 \mathrm{~mm}$ de es. pessura) sobre a qual se apoiavam os corpos de prova, desde à sua. obtenção até às posteriores mensura. ções de alteracões dimensionais.

"B" = A base acima referida ("A") era "emoldurada" por uma placa de acrílico de $3 \mathrm{~mm}$ de espes. sura, tendo em sua parte central uma abertura retangular com dimensões levemente maiores que as bases "A". A placa possuía duas guías de refe. rência " $b_{1}$ " e " $b_{2}$ ", que servíam para posicionar, corretamente, as partes "C" e "D".

"C e D" = Duas placas de acrilico, as quais, unidas e posicionadas através das guías de referência " $b_{1}$ " e " $b_{2}$ ", possuiam 1 em sua porção central uma abertura retangular de $56 \mathrm{~mm}$ por $19 \mathrm{~mm}$. A espessura des. tas duas placas era de $2,9 \mathrm{~mm}$. Estas medidas constituíam as dimensões dos futuros corpos de prova.

"E" = Uma p l a c a retangular $(30 / 60 \mathrm{~mm})$ constituida de 2 lâminas de acrílico, coladas uma na outra. Uma lâmina tinha $3 \mathrm{~mm}$ de espessura (acrílico mais duro) e a outra lamina tinha $1 \mathrm{~mm}$ de espessura (acrí. lico menos duro). Na superfície desta lamina, à distância de $43,874 \mathrm{~mm}$, existíam duas marcas de referência (depressões circulares) semelhantes a penetrações do tipo Brinell.

0 conjunt $_{o}$ das cinco partes, mon. tado, pode ser observado na Fig. de No 2. 

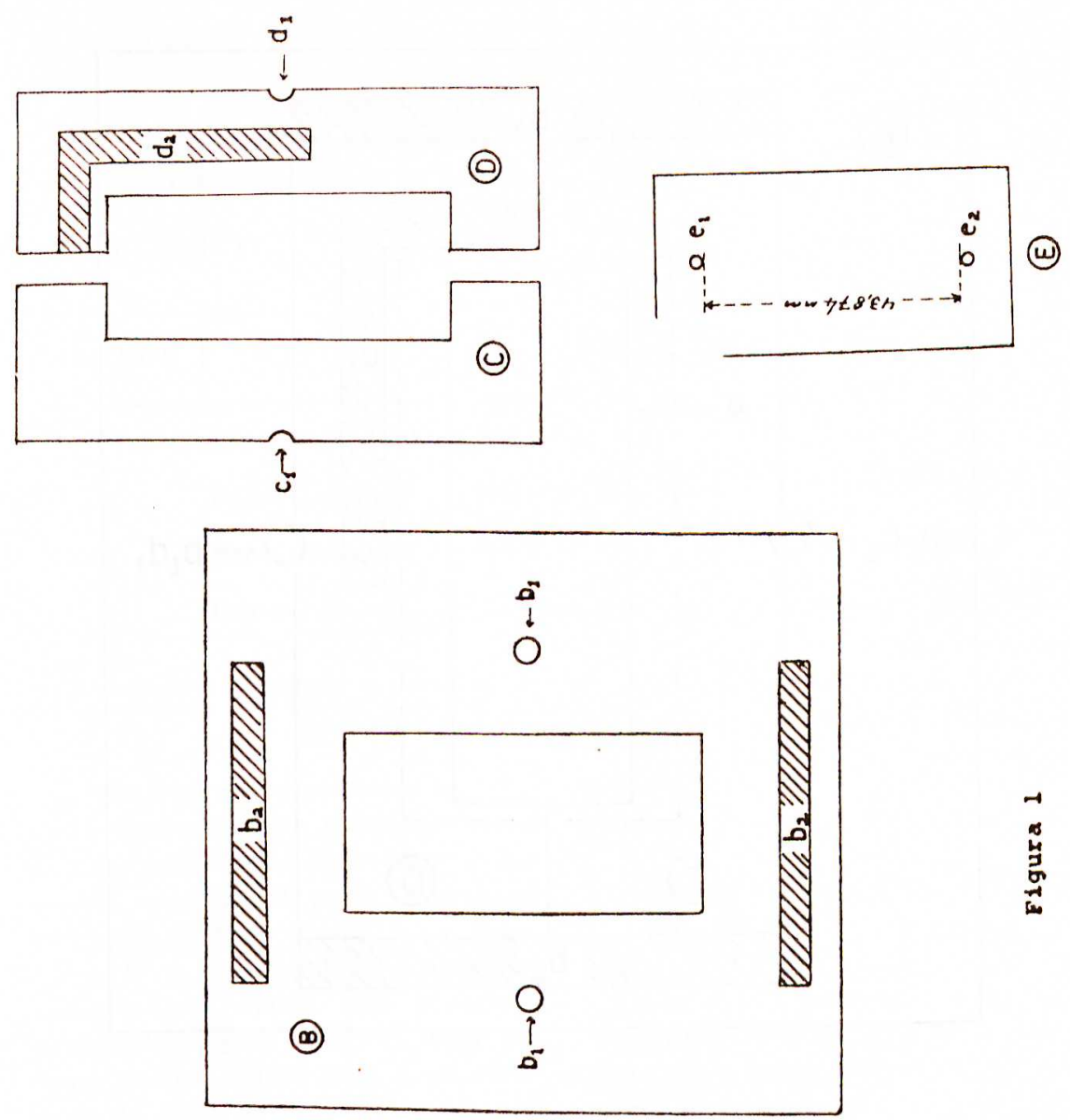

苟

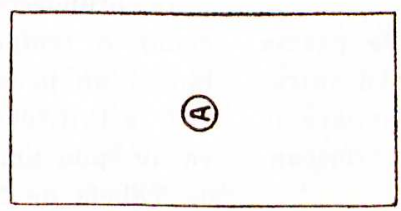

R. FAC. ODONT. P. A. 


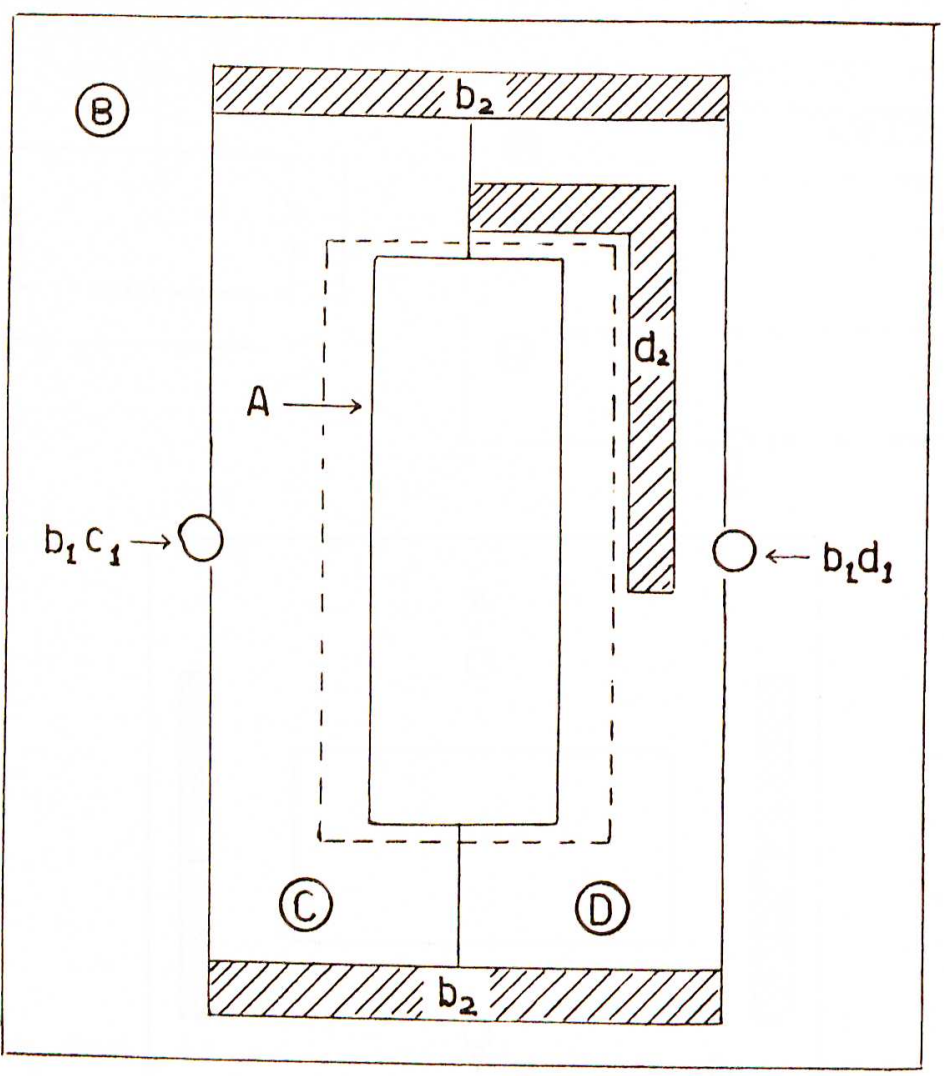

Figura 2

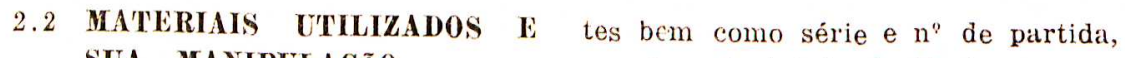
SUA MANIPULAC̄̃o ccnstam da tabela de $\mathrm{N}$ ! 1 .

As proporções ZnO/Eugenól, bem

Utilizaram.se 4 marcas de pastas zincoenólicas, comumente encontradas em nosso mercado de artigos o. dontológicos, cujos nomes, fabrican. como o tempo de espatulação e o tempo de permanência na Estufa a $37^{\circ} \mathrm{C}$ e U.R. $100 \%$, dos corpos de prova de cada uma das pastas, constam da Tabela de No 2. 
Tanto a proporção das pastas, bem como o seu tempo de espatulação, era os indicados pelos respectivos fabri. cantes. A indicação de proporção dizía comprimentos iguais de ambas as pastas. Em ensaios pilôto verifi. camos que necessitavamos, aproximadamente de $8 \mathrm{~cm}$ de cada uma das pastas.

TABELA No 1

\begin{tabular}{|l|l|l|l}
\hline LEGENDA & MA'TERIAL & FABRICANTE & Série/No Partida \\
\hline
\end{tabular}

$\begin{array}{lllr}\text { A } & \text { Pasta S. S. White S. S. White Art. Dent. S. A. } & 75.5458 / 300179 \\ \text { B } & \text { Pasta Luralite } & \text { Kerr Manufacturing Co. } & 3604 / 09157 \\ \text { C } & \text { Pasta Equalizing } & \text { Kerr Manufacturing Co. } & 3604 / 00142 \\ \text { D } & \text { Pasta Lysanda } & \text { Laboratórios Lysanda Ltda. } & \text { não consta }\end{array}$

- Imoportado e embalado p/Kerr Ind. e Comércio Ltda. Rua Antonio dos Santos Neto, 42, SP

o material correspondente às referidas dimensões, era pesado e face à distinta composição de cada uma das pastas, resultaram pesos diferentes, conforme se pode observar na Tabe. $1 a$ de $\mathrm{N}^{\circ} 2$. A proporção consequentemente era obtida por pesagens, utilizando-se para este fim, uma balança Metler P.1200 com a sensibili. dade de 0,001 $\mathrm{g}$.

As pastas eram espatuladas sobre uma lage de vidro, através de uma espátula de aço-inoxidável, semi.fle. xivel, durante o tempo indicado pelo respectivo fabricante, permitindo. se obter uma mistura homogênea e de colaração uniforme.

Os espaços de tempo de "permanência na estufa", correspondiam ao tempo indicado pelo fabricante, durante o qual os materiais deveríam permanecer na boca dos pacientes, antes de sua remoç̃o. Procurou-se destarte, simular as condições bu. cais por ocasião de uma moldagem.

\subsection{ELABORAÇ̃̃ DOS CORPOS DE PROVA}

Antes da "montagem" da matriz, os bordos laterais da abertura da parte “ $\mathrm{B} \gg \gg$ foram revestidos, internamente, com papel celofane. Isto era recessário devido à grande adesividade e pegajosidade do material.

A parte superior da placa "A" era revestida com fita durex, perfeitamen. te lisa e plana.

A. parte superior da placa "E", onde se encontravam as marcas, era revestida cuidadosamente com uma folha de estanho, (Globe-Extra Tough pure Tin.Foil $\mathrm{N}^{\circ} 10$, S. S. White Dent. Manf. Co.)

Montada a matriz, exceto a parte "E", após a manipulação do mate. 
rial, colocava-se a mistura no inte. rior da matriz, com leve excesso. Imediatamente comprimia.se o mate. rial com a placa " $E$ ", mantendo-se a pressão sob carga constante $(1.700$ g) até ao momento da posterior re. mocão da estufa.

O conjunto era levado à estufa, 2 minutos após o início da espatulação, lá permanencendo pelos espaços recomendados pelos fabricantes para permanencia $d_{o}$ material de moldagem na boca do paciente. (veja Tab. de $N^{\circ} 2$ )

\subsection{PROCEDIMENTOS DAS MEN. SURAÇ̃ES}

Após a retirada do conjunto da estufa, removia-se a placa "E"; remo- viam-se os pinos retentores " $b_{1}$ ", permitindo tal operação, a retirada das placas " $\mathrm{C}$ " e " $\mathrm{D}$ " deslisando.as paralelamente às barras guias " $b$ ". Retirava-se então a placa " $\mathrm{B} » »$, permanecendo o corpo de prova aderindo a placa "A".

Removia-se a folha de estanho, po. dendo-se observar agora na superfície do C. P. as duas marcas na forma de "calotas".

Imediatamente levava-se o C. P. apoiado na placa "A", ao microscópio de mensuração (Gaertner, sensibilidade 0,002 mm) e media.se a distância entre as marcas de referência, toman. do como base, as tangentes internas do círculos.

TABELA No 2

\begin{tabular}{|c|c|c|c|c|}
\hline \multirow[b]{2}{*}{ MAETIAL } & \multicolumn{2}{|c|}{ PROPORCXO DE PESO } & \multirow[b]{2}{*}{$\begin{array}{l}\text { TWMPO } \\
\text { DE ESPA } \\
\text { DE ESPA- } \\
\text { LACAO } \\
\text { (segundos) }\end{array}$} & \multirow[b]{2}{*}{\begin{tabular}{|c} 
TEMPO DE PERMANENCIA \\
DO C.P. NA ESTUHA A \\
$37 \%$ E 100\% DE U.R. \\
(minutos)
\end{tabular}} \\
\hline & $\begin{array}{c}\text { OXIDO } \\
\text { DE } \\
\text { zINCo } \\
\text { (garams) } \\
\end{array}$ & $\begin{array}{c}\text { EUGENOL } \\
\text { (gramas) }\end{array}$ & & \\
\hline $\mathbf{A}$ & 6 & 5,5 & 45 & 5 \\
\hline $\mathbf{B}$ & 14,5 & 3,5 & 39 & 3,30 \\
\hline $\mathrm{C}$ & 9 & 3 & 90 & 3,30 \\
\hline $\mathrm{D}$ & 6 & 5,5 & 45 & 3 \\
\hline
\end{tabular}

A primeira leitura era feita imediatamente após a remocão dos C. P's. da estufa (5 minutos) e as leituras seguintes aos 30, 45, 60 minutos, sen. do a última leitura após decorridas 24 horas.
Havia sido fixada à base do mi. croscópio, uma guia de referência, que permitia o posicionamento da placa "A" com o C. P., de maneira precisa e sem mínima perda $d_{e}$ tem. po. 


\subsection{CALCULOS DOS PERCEN. TUAIS DAS ALTERACOEES DI. MENSIONAIS}

Os valores para a porcentagem de alterações dimensionais eram calcu. lados a partir da seguinte fórmula:

$$
\frac{\mathrm{A}-\mathrm{B}}{\mathrm{C}} \times 100=\% \underset{\substack{\text { Alteração di. } \\ \text { mensional }}}{\mathrm{c}}
$$

em que: "A" era considerada como a primeira mensuração, realizada logo após a remoção do C. P. da estufa

"B" eram as leituras adicio. nais, consideradas individu. almente, realizadas nas ida. des constantes da Tabela de $\mathrm{N}^{\circ} 3$

"C" era a distância existente entre as duas marcas na matriz, e que era, precisa. mente, de $43,874 \mathrm{~mm}$.

Todos os resultados apresentados, se constituem da média de no míni. mo cinco (5) ensaios e, os resultados foram submetidos à análise estatística (teste "F" e teste " $t$ ") para obter. mos a indicação de seus niveis de significância. (Tabela de No 3 e 4 )

\section{RESULTADOS OBTIDOS}

Os resultados obtidos podem ser observados através dos Gráficos $G_{1}$, $\mathrm{G}_{2}, \mathrm{G}_{3}$ e $\mathrm{G}_{4}$ bem como através das Tabelas de $N^{\circ} 3$ e 4 .

3.1 Segundo se pode observar, con. forme os Gráficos $G_{1}$ e $G_{2}$, todos os materiais, exceto o material $\mathrm{D}$, apresentaram valores negativos, ou seja, valores correspondentes à contração. Com a idade de 24 horas, no entanto, também o material $\mathrm{D}$ apresentava. uma ligeira contração.

3.2 Analisando a significância entre as diferenças, conforme a idade dos corpos de prova, verificamos o seguinte:

\section{2 .130 minutos $\left(\mathrm{G}_{3}\right)$}

$$
\begin{aligned}
& .05=2,306 \\
& \mathrm{t}^{8}= \\
& .01=3,355 \\
& .05=0,055 \\
& .01=0,079
\end{aligned}
$$

todas as diferenças são altamento significativas, exceto a dife. renca entre os materiais $A$ e $B$ $\left(x_{1} \in x_{2}\right)$ que é apenas significativa.

\subsubsection{5 minutos $\left(\mathrm{G}_{3}\right)$}

$$
t_{\text {g }}=\begin{aligned}
& .05=2,306 \\
& .01=3,355
\end{aligned}
$$

$$
\text { DMS }=\begin{aligned}
& .05=0,041 \\
& .01=0,060
\end{aligned}
$$

todas as diferenças são altamente significativas exceto a diferen. ça entre os materiais A e B ( $x_{1}$ e $x_{2}$ ) que não é significativa. 
$3.2 .3 \quad 60$ minutos $\left(\mathrm{G}_{4}\right)$

$$
\begin{gathered}
\mathrm{t}_{8}=.05=2,306 \\
.01=3,355 \\
.05=0,035 \\
\text { DMS }=.01=0,051
\end{gathered}
$$

todas as diferenças são altamen. te significativas.

\section{2 .424 horas $\left(\mathrm{G}_{4}\right)$}

$$
\mathrm{t}_{\text {8 }}=\begin{array}{r}
.05=2,306 \\
.01=3,355
\end{array}
$$

$$
\text { DMS }=\begin{aligned}
& .05=0,083 \\
& .01=0,121
\end{aligned}
$$

todas as diferenças são altamente significativas, exceto a dife. renca entre os materiais $A$ e C $\left(x_{1}\right.$ e $\left.x_{3}\right)$ que não é significativa.

3.3 Conforme se pode observar nas Tabelas de $N$ ? 3 e 4 , os maiores

\begin{tabular}{|c|c|c|c|c|c|c|}
\hline \multirow{3}{*}{ Material } & \multicolumn{3}{|c|}{30 Minutos } & \multicolumn{3}{|c|}{45 Minutos } \\
\hline & Médias & s & $s^{2}$ & Médias & $\mathrm{s}$ & $s^{2}$ \\
\hline & $\%$ & $\begin{array}{l}\text { *Expo. } \\
\text { ente-3 }\end{array}$ & $\begin{array}{l}{ }^{*} \text { Expo. } \\
\text { ente-3 }\end{array}$ & $\%$ & $\begin{array}{l}{ }^{*} \text { Expo. } \\
\text { ente-3 }\end{array}$ & $\begin{array}{l}\text { *Expo. } \\
\text { onte-3 }\end{array}$ \\
\hline $\mathrm{X}_{1}^{* * *}$ & $.0,056$ & 21,753 & 0,473 & $.0,043$ & 35,223 & 1,241 \\
\hline$x_{2}+$ & $.0,041$ & 32,484 & 1,055 & $.0,041$ & 32,081 & 1,029 \\
\hline$x_{3}++$ & $.0,023$ & 14,195 & 0,201 & $.0,018$ & 5,550 & 0,031 \\
\hline$x_{4} £$ & $+0,040$ & 62,276 & 3,878 & $+0,039$ & 57,782 & 3,339 \\
\hline
\end{tabular}
valores de Variância (variabilida. de dentro do experimento) são os apresentados pelo material D $\left(x_{4}\right)$ seguidos, em ordem decrescente, pelos materiais $B\left(x_{2}\right)$, A $\left(\mathrm{x}_{1}\right)$ e $\mathrm{C}\left(\mathrm{x}_{3}\right)$

Tabela $n^{0} 3$

Avaliação Estatística dog Materiais nas Diferentes Idades

Idade dos Corpos de Prova

* o expoenter 3 para os valores destas colunas, não somente reduz o número de dígitos dos resultados originais, como permite cotejá-los. Ex.: $21,753^{-3}=0,021753$.

* Pasta S.S. White +Pasta Luralite-Kerr + +Pasta Equalizing .Kerr f. Pasta Lysanda. 
TABELA 4

Avaliação Estatística dos Materiais nas Diferentes Idades

\begin{tabular}{l|c|c|c|c|c|c}
\hline \multirow{2}{*}{ Marcas } & \multicolumn{3}{c}{ Idade dos Corpos de Prova } \\
\cline { 2 - 7 } & Médias & $\mathrm{s}$ & $\mathrm{s}^{2}$ & \multicolumn{3}{c}{24 Horas } \\
\cline { 2 - 7 } & $\%$ & $\begin{array}{c}* \text { Expo. } \\
\text { ente-3 }\end{array}$ & $\begin{array}{c}* \text { Expo- } \\
\text { ente-3 }\end{array}$ & $\%$ & $\begin{array}{c}{ }^{*} \text { Expo. } \\
\text { ente-3 }\end{array}$ & $\begin{array}{c}{ }^{*} \text { Expo- } \\
\text { ente-3 }\end{array}$ \\
\hline $\mathrm{X}_{1}{ }^{* *}$ & $-0,065$ & 39,443 & 1,556 & $-0,047$ & 13,217 & 0,175 \\
$\mathrm{X}_{2}+$ & $-0,050$ & 91,742 & 8,416 & $+0,102$ & 54,048 & 2,921 \\
$\mathrm{X}_{3}++$ & $-0,016$ & 7.733 & 0,060 & $-0,042$ & 25,961 & 0,674 \\
$\mathrm{X}_{4} £$ & $+0,028$ & 49,659 & 2,466 & $-0,003$ & 97,514 & 9,509 \\
\hline
\end{tabular}

* O expoente-3 para os valores destas colunas, não somente reduz o núme. ro de digitos dos resultados orginais, como permite aqui, melhor cote. já.los. Ex.: $39,443^{-3}=0,039443$.

** Pasta S. S. White + Pasta Luralite - Kerr + + Pasta Equilizing - Kerr f Pasta Lysanda.

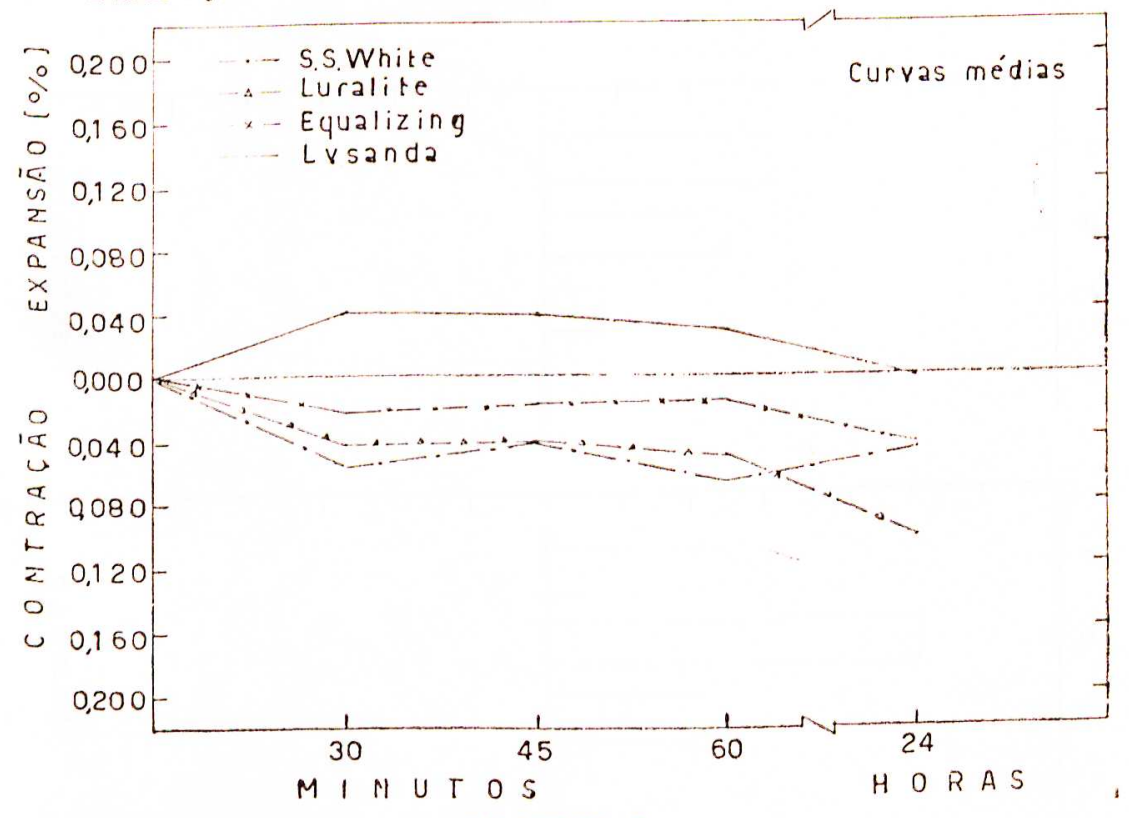

GRÁFICO I 

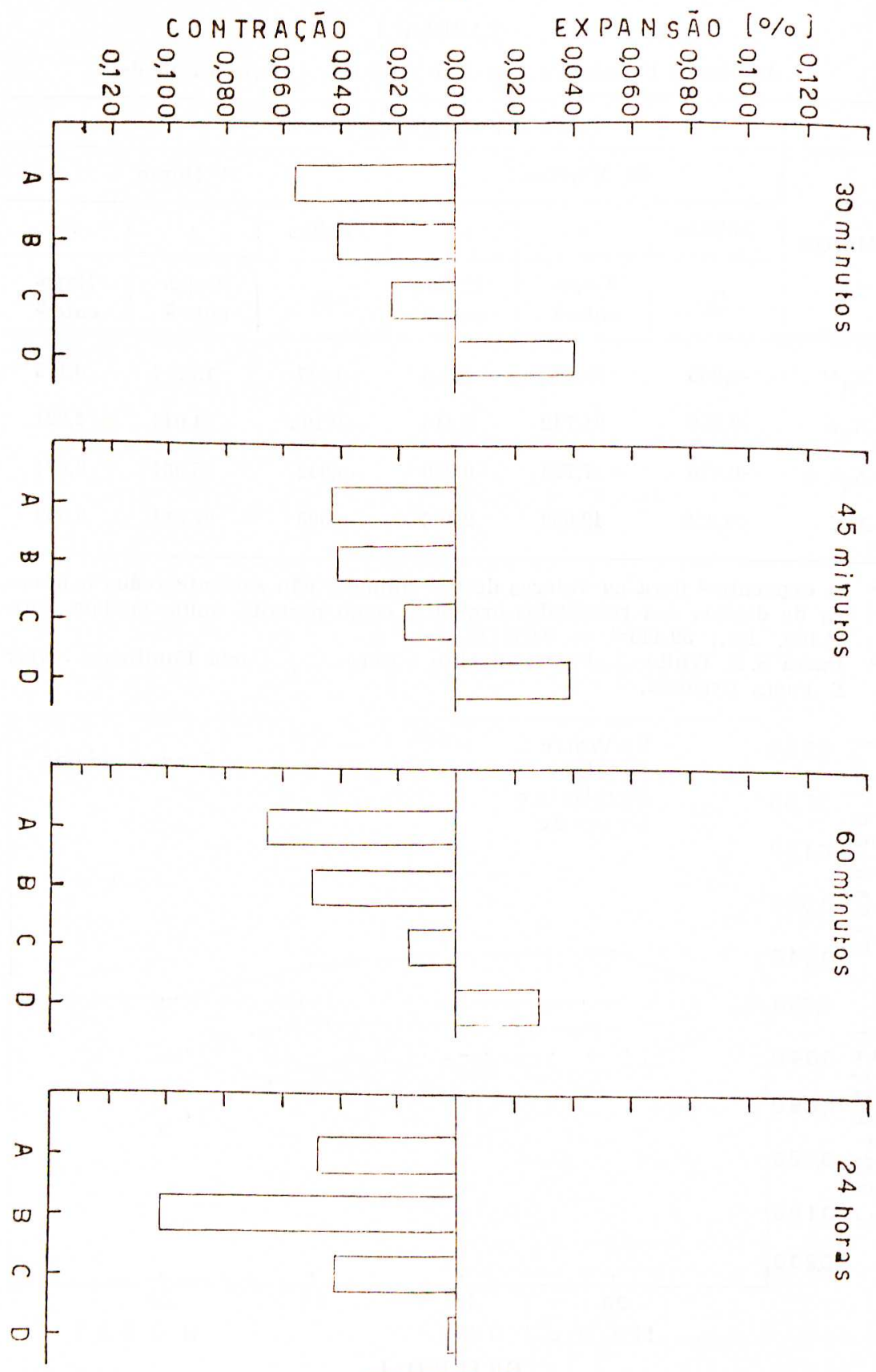

GRÄFICO II

R. FAC. ODONT. P. A. 


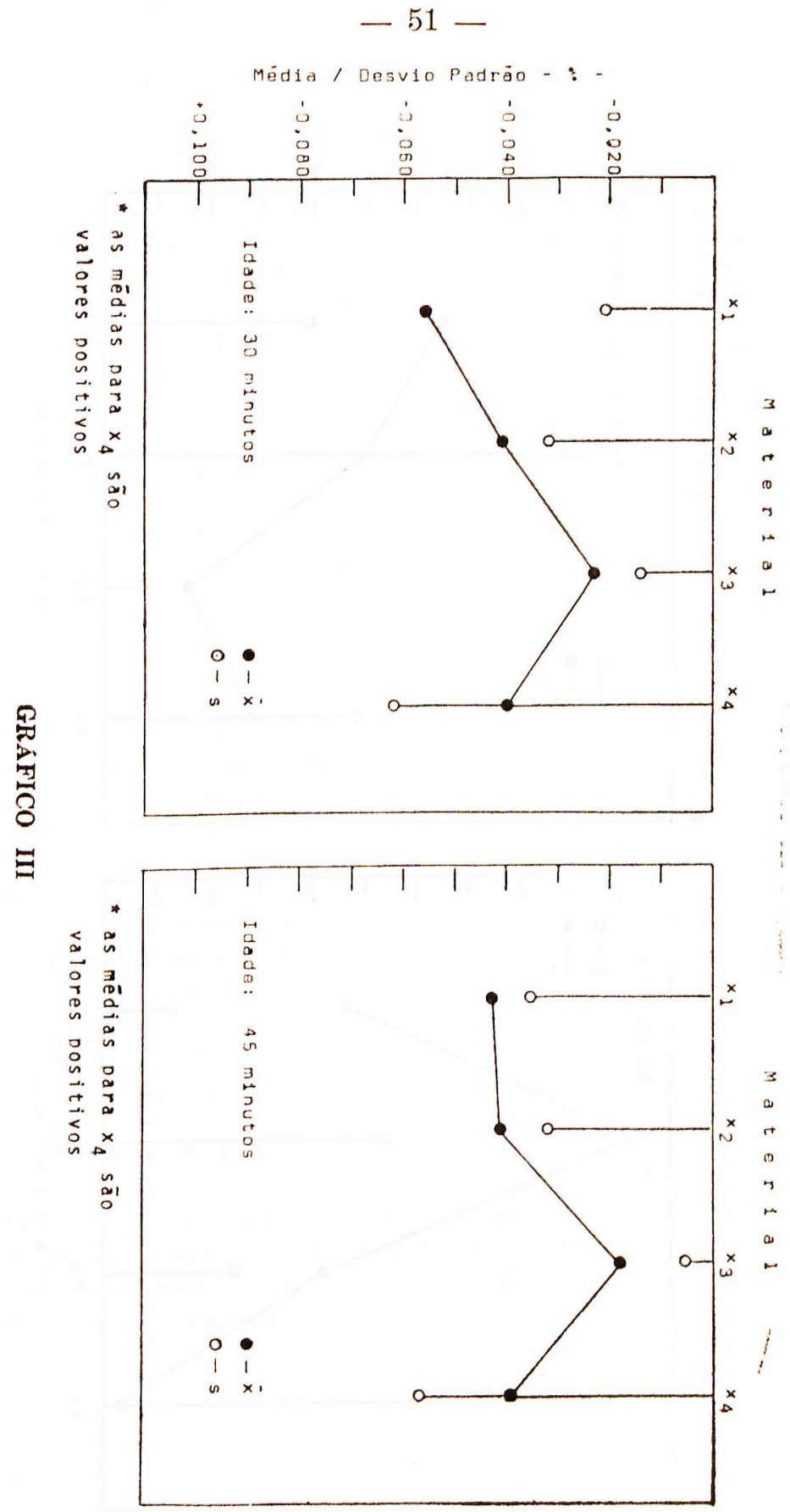

R. FAC, ODONT. P, A,

$18 / 19 / 20 ; \quad 39-55,1976 / 8$ 


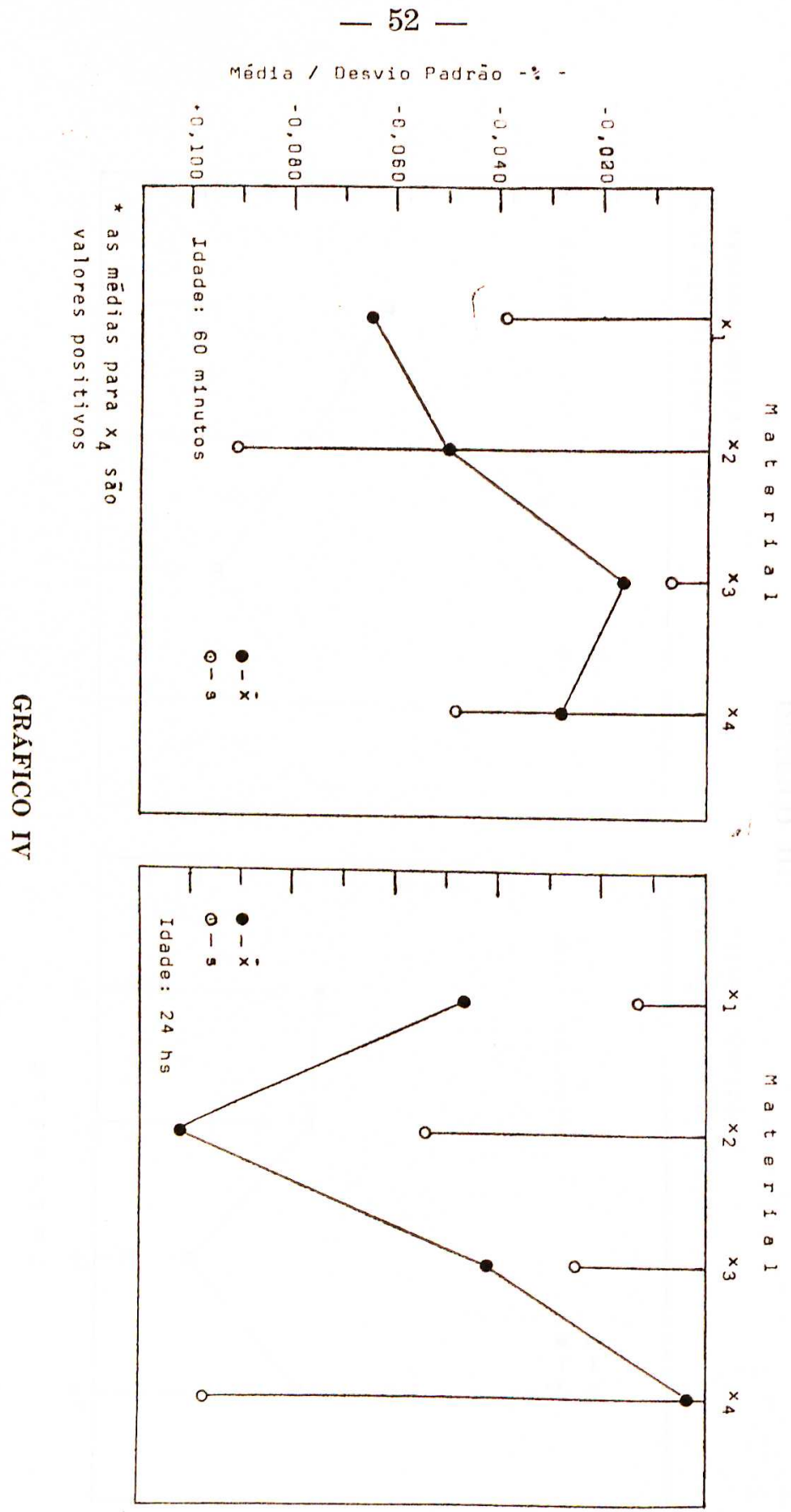

R. FAC. ODONT. P. A.

$18 / 19 / 20 ： 39-55,1976 / 8$ 


\section{CONCLUSÕES}

As pastas de óxido de zinco.euge. nól por nós analisadas apresentaram alterações dimensionais por ocasião de seu endurecimento, cujos valores iam desde $0,003 \%$ até $0,102 \%$.

o novo método para a determina. cão de alterações dimensionais que apresentamos comprovara sua repro. dutibilidade em relação à mercapta. nas e siliconas.

A mesma reprodutibilidade, no en. tanto, não se verificou em relação a pastas de óxido de zinco-eugenól, conforme ficou evidenciado através dos elevados valores de desvio pa. drão e variância.

Sugerese que, durante a armaze. nagem destes materiais, esteja ocorrendo a sedimentação dos componen. tes de maior densidade, havendo em conseqüência o desequilíbrio da com. posição.

o acondicionamento das pastas po. deria ser a semelhança das resinas compostas, cabendo ao dentista a homogeneização de cada uma delas antes de efetuar a mistura de ambas, em proporções indicadas.

Este assunto será motivo de uma próxima investigạão.

\section{S U M M A R Y}

After an extensive review of the literature, regarding zinc-oxide/eugenol impression pastes, we selected 20 of the most representatives as our references. Trough personal information of several of the investigator's it was discovered that dimensional changes of theese materials is one of the most difficult properties to be measured. A new method was deve. loped to measure dimensional changes of 4 (four) of the most widely used zinc-oxide/eugenol impression materials in Brazil.

The results, presented through several graphs and tables showed that dimensional changes varied from $0,003 \%$, values which may probably be considered negligible from a clini. cal point of view.

We noticed, however, high values for standard deviation and variance which indicate the high variability within the experiments.

Those values were not found when we used the same method with mercaptan and silicone impression mate. rials, in which the measurement of dimensional changes was highly re. producible.

One hypothesis (which we intend to investigate in a later research) is that, during storage, a sedimenta. tion could occur, of the components of greater density!

Consequently ther might result a change in composition, independent of the method used to establishe the proportion of the two pastes, be it by wheight or measurement of lenght, which could be the cause of va. riability of the composition of each mixture! 


\section{REFERÊNCIAS BHBLIOGRÁFICAS}

1. ASGAR, K. \& PEYTON, F. A. Physical properties of corrective impres. sion pastes, J. Pros. Dent. 4(4):555-567 Jul 1954

2. ASGAR, K. Physical properties of zinc oxide - eugenol impression pastes. J. D. Res. 33:693 (Abstr. Oct. 1954

3. BRAUER, G. M. et alii. The reaction of metal oxides with o.ethoxiben. zóic acid and other chelating agents. J. D. Res. 37(3):547.560 June 1958

4. COUNCIL FOR DENTAL RESEARCH. Council aproves A. D. A. Specification N: 16 for impression paste - zinc oxide - eugenol type. JADA (63) (1) : 164.167 Jul. 1961

5. COPELAND, H. I. et alii. Setting reaction of zinc oxide and eugenol. J. Res. of NBS b5(3):133.138 Set. 1955

6. DONNISON, JOAN A. Zinc oxide eugenol impression pastes. Austr. D. J. $5(5): 262.263$ Oct. 1960

7. HEMPTON, J. B. \& BEVAN, E. M. Proportioning zinc oxide - eugenol impression paste. Austr. D. J. 9:186.190 Jun. 1964

8. HIGASHI, S. \& YAJIMA, T. General researches for the impression ma. terial in the marquet. Part XI Bull. T'okyo Med. Dent. Univ. 7(4):623. 631. Dec. 1960

9. MOLNAR, E. J. \& SKINNER, E. W. A study of the zinc oxide-rosin ce. ments. I. Some variables which affect the hardening time. JADA 29 (1.6): Jan.Jun. 1942

10. MYers, G. E. \& PEYTON, F. A. Physical propoerties of the zinc oxi. de.engenol impression pastes. J. D. Res. 40(1):39.48 Jan.Feb. 1961

11. PHILLIPS, R. W. Review of impression materials. Internat. D. J.7:701717 Set. 1954

12. PINTO, F. E. Compuestos zinquenólicos para impressiones. Rev. O. Arg. 52:263.269 Jul. 1964

13. ROSS, R. A. Zinc oxide impression pastes. JADA 21:2029.2032 Nov. 1934

14. RUHNKE, L. A. \& GUERRINI, R. Influencia de solventes organicos

R. FAC. ODONT. P. A. $18 / 19 / 20: \quad 39-55,1976 / 8$ 
nas características físicas das pastas zincoenólicas. I. Influencia do xi. 1ol. An Fac. Odont. Piracicaba 1:37-44 1958

15. SKINNER, E. W.; COOPLR, E. N. \& ZIEHM, H. W. Some physical pro. perties of zinc oxide - eugenol impression pastes. MIDA 41(4):449. 455 Oct. 1950

16. STAFFORD, G. D. \& WILSON, H. J. A study of the zinc oxide - euge. nol type impression materials. Brit. I). J. 117:108-112 Aug. 1964

17. TYAS, M. J. \& WILson, H. J. Properties of zinc oxide - eugenol im. pression pastes. Irrit. D. J. 129(10):461.466 Nov. 1970

18. VIetra, D. F. Pastas zincoenólicas. An. Fac. Furm. Odont. U. S. P. 10:329.347 1952

19. VIEIRA, D. F. \& CLSAR, A. Influencia da adicão de breu, acetato de zinco e óleo de oliva, sobre o tempo de prêsa e a consistência de misturas à base de óxido de zinco e eugenól. R. Fac. Odont. S. Paulo $5(2)$ : 95.104 Abr.Jun 1967

20. WILSON, H. J. A method of assessing characteristics of impression materials. Brit. D. J. $117(12): 536.540$ Dec. 1964 